\title{
BMJ Open Clinical pneumonia in the hospitalised child in Malawi in the post- pneumococcal conjugate vaccine era: a prospective hospital-based observational study
}

Pui-Ying Iroh Tam (D) , ,2,3 James Chirombo, ${ }^{4}$ Marc Henrion, ${ }^{2,4}$ Laura Newberry, ${ }^{1}$ Ivan Mambule, ${ }^{3,5}$ Dean Everett, ${ }^{3,6}$ Charles Mwansambo, ${ }^{7}$ Nigel Cunliffe, ${ }^{5}$ Neil French, ${ }^{8}$ Robert S Heyderman, ${ }^{9}$ Naor Bar-Zeev, ${ }^{1,3,9,10}$ For the VacSurv Consortium

To cite: Iroh Tam P-Y, Chirombo J, Henrion M, et al. Clinical pneumonia in the hospitalised child in Malawi in the post-pneumococcal conjugate vaccine era: a prospective hospital-based observational study. BMJ Open 2022;12:e050188. doi:10.1136/ bmjopen-2021-050188

\section{- Prepublication history and} additional supplemental material for this paper are available online. To view these files, please visit the journal online (http://dx.doi.org/10.1136/ bmjopen-2021-050188).

$\mathrm{PI}$ and $\mathrm{JC}$ are joint first authors.

Received 12 February 2021 Accepted 26 January 2022
Check for updates

(C) Author(s) (or their employer(s)) 2022. Re-use permitted under CC BY. Published by BMJ.

For numbered affiliations see end of article.

Correspondence to Dr Pui-Ying Iroh Tam; irohtam@mlw.mw

\section{ABSTRACT}

Objective Assess characteristics of clinical pneumonia after introduction of pneumococcal conjugate vaccine (PCV), by HIV exposure status, in children hospitalised in a governmental hospital in Malawi.

Methods and findings We evaluated 1139 children $\leq 5$ years old hospitalised with clinical pneumonia: 101 HIV-exposed, uninfected (HEU) and 1038 HIV-unexposed, uninfected (HUU). Median age was 11 months (IQR 6-20), $59 \%$ were male, median mid-upper arm circumference (MUAC) was $14 \mathrm{~cm}$ (IQR 13-15) and mean weight-forheight $z$ score was $-0.7( \pm 2.5)$. The highest Respiratory Index of Severity in Children (RISC) scores were allocated to $10.4 \%$ of the overall cohort. Only $45.7 \%$ had fever, and $37.2 \%$ had at least one danger sign at presentation. The most common clinical features were crackles $(54.7 \%)$, nasal flaring (53.5\%) and lower chest wall indrawing (53.2\%). Compared with HUU, HEU children were significantly younger ( 9 months vs 11 months), with lower mean birth weight $(2.8 \mathrm{~kg}$ vs $3.0 \mathrm{~kg})$ and MUAC $(13.6 \mathrm{~cm}$ vs $14.0 \mathrm{~cm})$, had higher prevalence of vomiting $(32.7 \%$ vs $22.0 \%$ ), tachypnoea ( $68.4 \%$ vs $49.8 \%$ ) and highest RISC scores (20.0\% vs $9.4 \%$ ). Five children died $(0.4 \%)$. However, clinical outcomes were similar for both groups. Conclusions In this post-PCV setting where prevalence of HIV and malnutrition is high, children hospitalised fulfilling the WHO Integrated Management of Childhood IIIness criteria for clinical pneumonia present with heterogeneous features. These vary by HIV exposure status but this does not influence either the frequency of danger signs or mortality. The poor performance of available severity scores in this population and the absence of more specific diagnostics hinder appropriate antimicrobial stewardship and the rational application of other interventions.

\section{INTRODUCTION}

Pneumonia is the leading cause of morbidity and mortality in children globally. This disease is of particular importance in lowincome countries such as Malawi, where

\section{Strengths and limitations of this study}

- We evaluated over 1100 children hospitalised with pneumonia in a low-income country setting after introduction of pneumococcal conjugate vaccine (PCV).

- This observational cohort was nested within a prospective hospital-based study of PCV13 effectiveness.

- We assessed the demographic and clinical characteristics of clinical pneumonia patients and compared HIV-exposed, uninfected versus HIVunexposed, uninfected children and computed Respiratory Index of Severity in Children scores for severe pneumonia.

- This study was limited by the low mortality rate, small proportion of HIV-exposed infants with HIV status tested by PCR, the single-centre study, a substantial proportion of incomplete files and lack of recruitment of moribund children who died very soon after arrival into the study. However, this was a detailed prospective study conducted in a lowresource setting in the post-PCV era where robust diagnostic testing was available.

HIV prevalence among pregnant women is $10.8 \%{ }^{1}$ and where a high burden of malaria and malnutrition (stunting prevalence of $39 \%^{2}$ ) contributes to an under-5 mortality rate of 55 per 1000 live births. ${ }^{3}$

WHO Integrated Management of Childhood Illness (IMCI) guidelines for community and referral level management of children with pneumonia rely on clinical recognition of features of severe disease. WHO lists general danger signs, such as inability to drink, persistent vomiting, convulsions, lethargy or unconsciousness, or stridor in a calm child, which are clinical features used to 
identify children who should be treated in an inpatient setting and to receive injectable antibiotics. ${ }^{4}$ However, clinical features are non-specific and can reflect a variety of other infectious (eg, bronchiolitis, malaria, sepsis, tuberculosis, pneumocystis) and non-infectious (eg, asthma, lymphocytic interstitial pneumonitis, pulmonary Kaposi sarcoma) conditions. Inappropriate and overuse of antimicrobials in low resource settings where antimicrobial prescribing is based more on clinical features than objective diagnostic data has implications for antimicrobial stewardship in the post-pneumococcal conjugate vaccine $(\mathrm{PCV})$ era, particularly in settings where antimicrobial choice and supply is limited and antimicrobial resistance rates are rising. ${ }^{5}$

Few risk scoring systems to predict severe pneumonia exist that are adaptable to low-income countries with high HIV prevalence-Respiratory Index of Severity in Children-Malawi (RISC-Malawi) is the only score from children from a low-income country, and did not consider HIV status. Furthermore, although the alternative, RISC score does include a scoring system based on HIV infection, no studies have specifically evaluated whether HIV exposure status affects the presence and predictive value of clinical features for severe disease and case-fatality. As HIV exposed, uninfected (HEU) children constitute a growing population and, compared with HIV-unexposed, uninfected (HUU) remain vulnerable to more severe pneumonia, more often fail empiric pneumonia treatment, and have higher rates of hospitalisation and death than HUU children, ${ }^{6-9}$ this population is of growing clinical relevance. Our objectives were to describe the clinical features of children hospitalised with pneumonia, calculate risk scores for our population, and to determine whether differences in clinical characteristics and calculated risk scores exist by HIV exposure status.

\section{METHODS}

This observational cohort was nested within a prospective hospital-based study of 13-valent PCV effectiveness conducted at Queen Elizabeth Central Hospital between 2 April 2013 and 3 August 2016. Children born on or after 1 October 2012 (based on verbal and written documentation from parents on time since birth) and therefore eligible for PCV and admitted with a diagnosis of clinical pneumonia were enrolled (online supplemental table 1). Children were excluded if they had known oncological or congenital heart disease, were admitted $\geq 48$ hours before recruitment, were re-admitted to hospital within 14 days of a previous hospitalisation, or presented moribund with impending death. Study staff performed active case finding Monday-Friday during daytime in the emergency department and inpatient wards, and followed up study participants 6 weeks following hospital discharge with a home visit or phone call to confirm vital status.

With informed written parental consent, all enrolled children had baseline characteristics measured which included clinical, laboratory and sociodemographic variables. Oxygen saturation was measured with an appropriately sized sensor and a Nellcor pulse oximeter (Welch Allyn Spot Vital Signs Devices, Skaneateles Falls, New York, USA) in room air. Children with oxygen saturations $<90 \%$ in room air were given supplemental oxygen via nasal cannula using an oxygen concentrator. At clinician decision and if a free circuit was available, children with severe respiratory distress, signs of exhaustion or worsening hypoxaemia received bubble continuous positive airway pressure (Pumani, third Stone Design, San Rafael, California, USA). Blood was routinely collected for packed cell volume and malaria parasite thick film. Mothers and infants were tested for HIV according to the Malawi National HIV Guidelines..$^{10}$ A blood culture was obtained only when the admitting clinician suspected sepsis. Children with suspected meningitis, malaria or anaemia, were managed according to respective WHO and hospital treatment guidelines. ${ }^{11}$

\section{Patient and public involvement}

Patients or the public were not involved in the design, or conduct, or reporting, or dissemination plans of our research.

\section{Case definition}

Pneumonia was diagnosed clinically per WHO IMCI criteria.

\section{Statistical analysis}

We assessed the demographic and clinical characteristics of clinical pneumonia patients and compared HEU versus HUU children using Fisher's exact test, two-sample t-test or Wilcoxon rank-sum tests, respectively, for categorical, continuous normal and continuous skewed variables.

We computed RISC scores for severe pneumonia in our cohort, ${ }^{12-14}$ using the variables listed in table 1 . We had insufficient variables to compute modified RISC (mRISC) and RISC-Malawi scores. ${ }^{13}$

\section{RESULTS}

A total of 1660 children were recruited into the study, among whose files were incomplete for 521 . We included 1139 children in the analysis, of whom 101 were HEU and 1,038 HUU. The median age was 11 months (IQR 6.0-20.0), 59\% were male, and the median mid-upper

\begin{tabular}{lc}
\hline Table 1 & List of variables used to compute RISC scores \\
\hline RISC & Score \\
\hline Oxygen saturation $\leq 90 \%$ & 3 \\
Chest indrawing & 2 \\
Refusal to feed & 1 \\
Wheezing & -2 \\
WHO weight for age $z$-score $\leq-3$ & 2 \\
WHO weight for age $z$-score $-3 \leq \_z<-2$ & 1
\end{tabular}

RISC, Respiratory Index of Severity in Children. 
arm circumference (MUAC) was $14 \mathrm{~cm}$ (IQR 13-15) and mean weight-for-height $\mathrm{z}$ score $-0.7( \pm 2.5$; table 2 and online supplemental table 2).

In our cohort, no single clinical feature was present at enrolment in more than $55 \%$ of the population: the most common clinical features were crackles $(54.7 \%)$, nasal flaring $(53.5 \%)$ and lower chest wall indrawing (53.2\%). Less than half had fever (45.7\%), and at least one IMCI danger sign $(37.2 \%)$. The median oxygen saturation at presentation was $95 \%$ (IQR 89-98), with $25.4 \%$ having oxygen saturations below $90 \%$. Among this cohort, $33.9 \%$ received oxygen and $2.1 \%$ were placed on continuous positive airway pressure (CPAP). The highest RISC scores (3+) were allocated to $10.4 \%$ of the overall cohort, respectively. Five children died $(0.4 \%)$.

Ten per cent of children in our cohort were HEU, and notable differences between HEU and HUU were observed in several areas. In the HEU group, children were younger (median age 9 months (IQR 5.0-15.5) vs 11 months (IQR 6.0-20.0), $\mathrm{p}=0.02$ ), with lower birth weight (median $2.8 \mathrm{~kg}$ (IQR 1.8-3.2) vs $3.0 \mathrm{~kg}$ (IQR 2.4-3.3), $\mathrm{p}=0.03$ ), lower weight-for-height $\mathrm{z}$ score (mean $-1.2 \pm 2.5 \mathrm{vs}$ $-0.6 \pm 2.5, \mathrm{p}<0.01$ ) and lower MUAC (median $13.6 \mathrm{~cm}$ (IQR 12.6-14.6) vs $14 \mathrm{~cm}$ (IQR 13-15), $\mathrm{p}<0.01$ ). Compared with the HUU group, HEU children had significantly higher prevalence of vomiting $(32.7 \%$ vs $22.0 \%, \mathrm{p}=0.02)$ and age-defined tachypnoea in infancy $(68.4 \%$ vs $49.8 \%$, $\mathrm{p}=0.02)$. More HEU children presented with at least one IMCI danger sign $(46.5 \%$ vs $36.3 \%, \mathrm{p}=0.05)$. We did not observe significant differences in respiratory symptoms and in proportions of children who died between groups, although HEU children received oxygen at a significantly higher rate than HUU children $(44.0 \%$ vs $32.9 \%$, $\mathrm{p}=0.04$ ). Clinical outcomes between the HEU and HUU groups were also similar (table 2). However, RISC scores were significantly different between HIV exposure status, with $20.0 \%$ of HEU with the highest scores, compared with $9.4 \%$ of HUU (figure 1). The risk score had a nonparametric distribution and many subjects scored 0 .

\section{DISCUSSION}

In our prospective cohort of children presenting with WHO IMCI criteria for clinical pneumonia in the post-PCV era, the most common clinical features were crackles, nasal flaring and lower chest wall indrawing. Despite the clinical diagnosis of pneumonia, features were heterogeneous. Thirty-four per cent had an oxygen requirement and $2.1 \%$ needed CPAP support, though with best available treatment, overall mortality was low-and possibly a reflection of improvements in child health overall as a result of antiretroviral and antimalarial therapies, vaccination and community management of nutrition. Ten per cent of children hospitalised with pneumonia were HEU and this group was significantly younger, with lower birth weight and anthropometric parameters. HEU children had a significantly higher prevalence of vomiting, age-defined tachypnoea in infancy, and higher RISC scores, compared with HUU children.

Though HEU children were significantly younger and had significantly lower MUAC than HUU children, and therefore, age and anthropometry are potential triage indicators for use in the clinical management of pneumonia, these differences were subtle and may not be useful for a clinician, who in low-resource settings make decisions primarily on clinical suspicion. WHO IMCI clinical criteria for pneumonia are non-specific, and based on presence of cough, fast and difficult breathing. The nonspecific nature of the diagnosis, which covers both infectious and non-infectious causes, is deliberately intended to increase sensitivity; however, as reflected in this study, this may have resulted in the term being used with a variety of features. Much research has been expended on developing pneumonia risk scores to identify children at risk of severe disease. However, the limitations of existing pneumonia risk scores for children in low-income and middle-income country settings ${ }^{15}$ including Pneumonia Etiology Research in Child Health (PERCH) ${ }^{16}$ Respiratory Syncytial Virus Network, ${ }^{17}$ RISC, ${ }^{14}$ mRISC $^{12}$ and RISC-Malawi, ${ }^{13}$ is that the predictive capacity is generally modest and, in the case of PERCH, was found to be not as discriminatory as the WHO danger signs. The risk scores we calculated in our cohort were not normally distributed and yielded surprisingly divergent results, indicating that they are limited to their study populations and are not generalisable.

In our cohort, the lack of predominating features of clinical pneumonia points to another possibility that, in post-PCV settings where HIV and chronic malnutrition rates are high (10\% and $39 \%$, respectively), children do not present with typical signs and symptoms. The difference in distribution of risk scores within our cohort highlights the fact that risk scores are populationspecific and cohort-specific. In our cohort a substantial proportion of children met criteria for severe acute malnutrition $(9.7 \%$ had weight-for-height $\mathrm{z}$ scores $\leq 3$; and $4.1 \%$ had MUAC $<11.5 \mathrm{~cm}$ ), indicating how widespread chronic malnutrition is in Malawi. This pervasive underlying comorbidity leads to subsequent issues with identification and management of disease; and may in part explain overuse of antimicrobials in this setting, one factor contributing to an increase in antimicrobial resistance.

Antimicrobial stewardship and infection control issues in low-resource settings have been particularly problematic among young children, with significant increases in antimicrobial resistance noted among young infants with bloodstream infections over a 20 -year period, ${ }^{5}$ and concerns over resistance rates in childhood pneumonia in sub-Saharan Africa. ${ }^{18}$ Challenges with diagnostic capacity and the limitations of clinical diagnosis hinder appropriate antimicrobial stewardship. ${ }^{19}$ Risk scores that are adequately discriminatory, therefore, present an opportunity for targeted interventions, including early aggressive support, as well as judicious allocation of resources. 
Open access

Table 2 Characteristics of children hospitalised with clinical pneumonia, stratified by HIV exposure status

\begin{tabular}{|c|c|c|c|c|}
\hline \multirow[b]{2}{*}{ Characteristics } & \multirow[b]{2}{*}{ All $(\mathrm{N}=1139)$} & \multicolumn{2}{|c|}{ Group } & \multirow[b]{2}{*}{$P$ value } \\
\hline & & HEU $(\mathrm{N}=101)$ & HUU ( $=1038)$ & \\
\hline \multicolumn{5}{|c|}{ Demographic and physiological characteristics } \\
\hline $\mathrm{N}$ & 1118 & 99 & 1019 & \\
\hline Median (IQR) & $11.0(6.0-20.0)$ & $9.0(5.0-15.5)$ & $11.0(6.0-20.0)$ & \\
\hline Male (\%) & $677(59.4)$ & $61(60.4)$ & $616(59.3)$ & \\
\hline Birth weight, kg & & & & $0.03^{*}$ \\
\hline $\mathrm{N}$ & 1117 & 97 & 1020 & \\
\hline Median (IQR) & $3.0(2.3-3.3)$ & $2.8(1.8-3.2)$ & $3.0(2.4-3.3)$ & \\
\hline MUAC, $\mathrm{cm}$ & & & & $<0.01^{*}$ \\
\hline $\mathrm{N}$ & 1129 & 101 & 1028 & \\
\hline Median (IQR) & $14.0(13.0-15.0)$ & $13.6(12.6-14.6)$ & $14.0(13.0-15.0)$ & \\
\hline Temperature & & & & $0.02^{*}$ \\
\hline $\mathrm{N}$ & 1133 & 100 & 1033 & \\
\hline Median (IQR) & $37.8(36.9-38.6)$ & $38.0(37.2-39.0)$ & $37.8(36.8-38.5)$ & \\
\hline Fever & & & & 0.06 \\
\hline $\mathrm{N}$ & 1133 & 100 & 1033 & \\
\hline Yes (\%) & $518(45.7)$ & $55(55.0)$ & $463(44.8)$ & \\
\hline Vomiting & & & & $0.02 \dagger$ \\
\hline $\mathrm{N}$ & 1137 & 101 & 1036 & \\
\hline Yes (\%) & $261(23.0)$ & $33(32.7)$ & $228(22.0)$ & \\
\hline At least one $\mathrm{IMCl}$ danger sign & & & & $0.05 \dagger$ \\
\hline $\mathrm{N}$ & 1139 & 101 & 1038 & \\
\hline Yes (\%) & $424(37.2)$ & $47(46.5)$ & $377(36.3)$ & \\
\hline \multicolumn{5}{|l|}{ Clinical features } \\
\hline Nasal flaring & & & & $0.92 \dagger$ \\
\hline $\mathrm{N}$ & 1137 & 101 & 1036 & \\
\hline Yes (\%) & $608(53.5)$ & $55(54.5)$ & $553(53.4)$ & \\
\hline Lower chest wall indrawing & & & & $1.00 \dagger$ \\
\hline $\mathrm{N}$ & 1137 & 101 & 1036 & \\
\hline Yes (\%) & $605(53.2)$ & $54(53.5)$ & $551(53.2)$ & \\
\hline Stridor & & & & $0.44 \dagger$ \\
\hline $\mathrm{N}$ & 1137 & 101 & 1036 & \\
\hline Yes (\%) & $22(1.9)$ & $3(3.0)$ & $19(1.8)$ & \\
\hline Age-defined tachypnoea & & & & $0.02 \dagger$ \\
\hline $\mathrm{N}$ & 666 & 57 & 609 & \\
\hline
\end{tabular}


Table 2 Continued

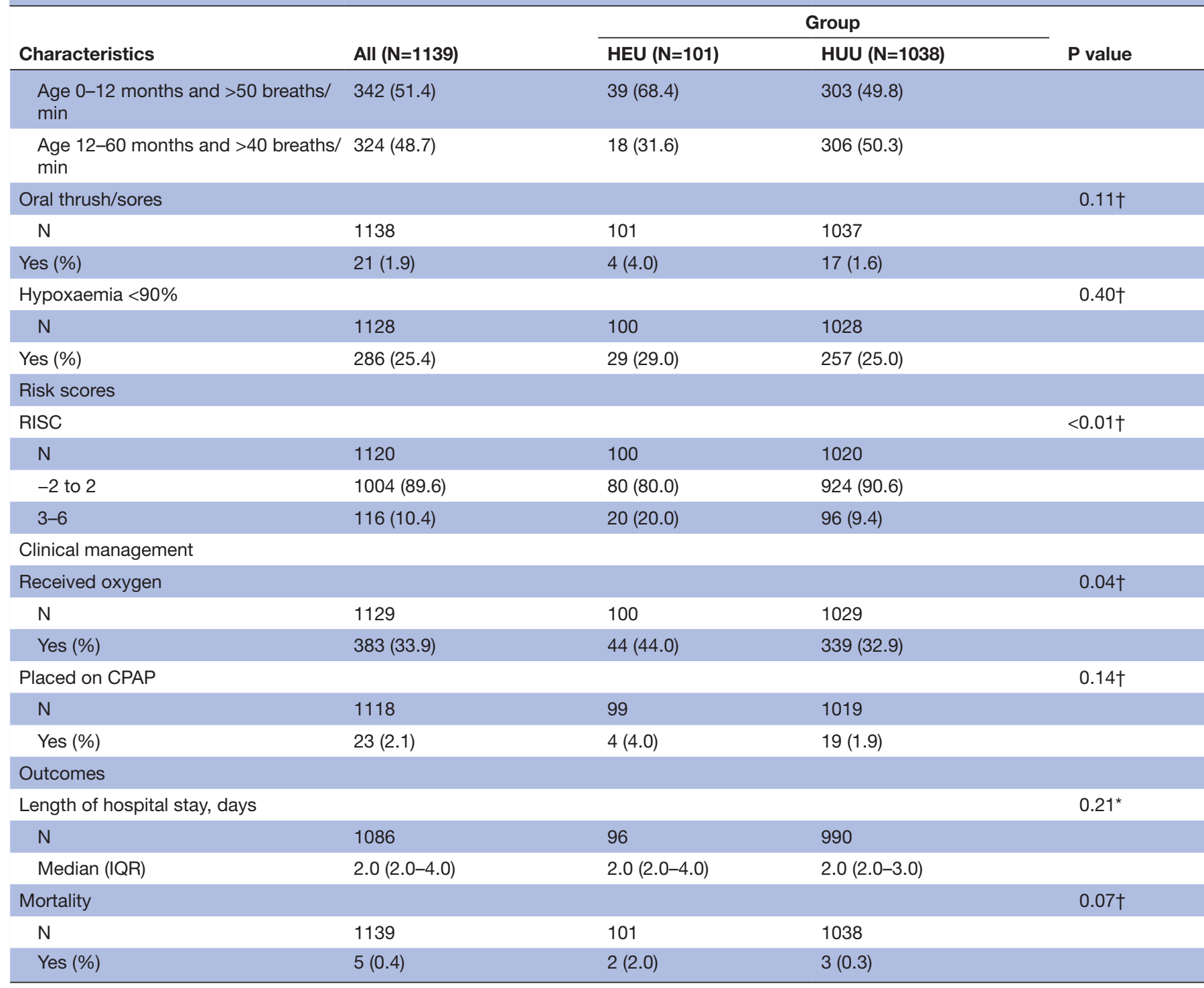

*Wilcoxon rank-sum test.

†Fisher's exact test.

fIndependent two sample t-test.

CPAP, continuous positive airway pressure; HEU, HIV-exposed, uninfected; HUU, HIV-unexposed, uninfected; IMCI, Integrated Management of

Childhood IIIness; IQR, interquartile range; MUAC, mid-upper arm circumference; RISC, Respiratory Index of Severity in Children.

This study has several limitations. Only a very small proportion of HIV-exposed infants had HIV status tested by PCR, as recommended by national guidelines. This was due to shortage of HIV counsellors, lack of testing reagents and reluctance of parents to obtain additional testing on their children. We did not recruit moribund children who died very soon after arrival, and therefore, we may have underestimated our fatality rate. Demographics may play into admissions at a government referral tertiary hospital, and therefore, there may be selection bias for sicker children to present. A substantial proportion of recruited participants had incomplete files and were therefore not included in the analysis. Due to small numbers, we were unable to calculate predictive values for severe outcomes. However, this was a detailed prospective study conducted in a low resource setting in the post-PCV era where robust diagnostic testing was available.

In conclusion, in this setting where prevalence of HIV and malnutrition is high, children hospitalised fulfilling the WHO IMCI criteria for clinical pneumonia in the post-PCV era present with heterogeneous features. These vary by HIV exposure status but this does not influence either the frequency of danger signs or mortality. The sample size limited our ability to calculate predictive scores; and the performance of available severity scores in this population and the absence of more specific diagnostics hinder appropriate antimicrobial stewardship and the rational application of other interventions. Further work in developing antimicrobial stewardship scores in 


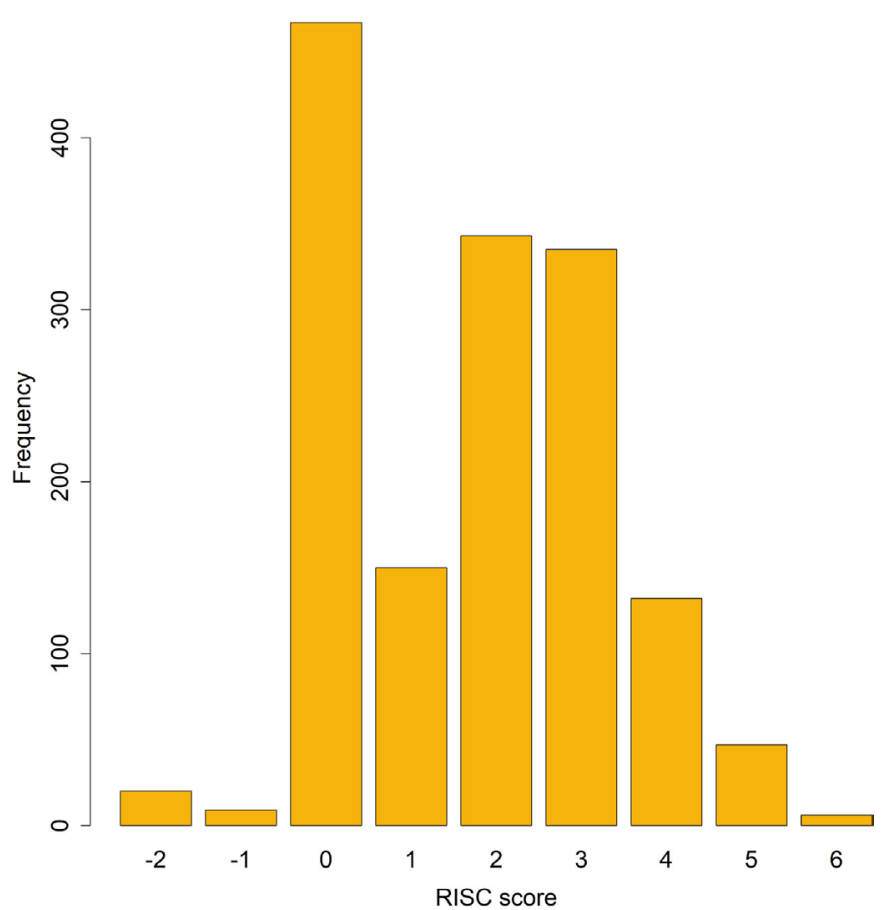

Figure 1 Summary of RISC scores computed from the data. RISC, Respiratory Index of Severity in Children.

resource-limited settings, in a period defined by rising rates of antimicrobial resistance, may be warranted.

\section{Author affiliations}

${ }^{1}$ Department of Paediatrics and Child Health, Queen Elizabeth Central Hospital, Blantyre, Malawi

${ }^{2}$ Department of Clinical Sciences, Liverpool School of Tropical Medicine, Liverpool, UK

${ }^{3}$ Paediatrics and Child Health Research Group, Malawi-Liverpool Wellcome Programme, Blantyre, Malawi

${ }^{4}$ Statistical Support Unit, Malawi-Liverpool Wellcome Programme, Blantyre, Malawi ${ }^{5}$ Clinical Infection, Microbiology and Immunology, University of Liverpool, Liverpool, UK

${ }^{6}$ Pathology and Infectious Diseases, Khalifa University, Abu Dhabi, UAE

${ }^{7}$ Government of Malawi Ministry of Health, Lilongwe, Malawi

${ }^{8}$ Centre for Global Vaccine Research, Institute of Infection and Global Health, University of Liverpool Faculty of Health and Life Sciences, Liverpool, UK ${ }^{9}$ Division of Infection and Immunity, University College London, London, UK ${ }^{10} \mathrm{Global}$ Disease Epidemiology and Control, Johns Hopkins University School of Public Health, Baltimore, Maryland, USA

Acknowledgements The authors wish to thank the collaborating members of the VacSurv Consortium (James Beard, Amelia C Crampin, Carina King, Sonia Lewycka, Hazzie Mvula, Tambosi Phiri, Miren Iturriza-Gomara, Osamu Nakagomi, Jennifer Verani, Cynthia Whitney).

Collaborators VacSurv Consortium: James Beard, Amelia C Crampin, Carina King, Sonia Lewycka, Hazzie Mvula, Tambosi Phiri, Miren Iturriza-Gomara, Osamu Nakagomi, Jennifer Verani, Cynthia Whitney.

Contributors Conceived and designed the research question: $\mathrm{PI}$ and NBZ; Conducted the statistical analysis: JC and MH; Managed the study: LN and IM; Developed and awarded funding for the study: DE, NC, CM, NF and RSH; Wrote the first draft of the manuscript: PI; Provided critical feedback on the manuscript: JC, $\mathrm{MH}, \mathrm{NC}, \mathrm{NF}, \mathrm{RSH}$ and NBZ; Reviewed and approved the final draft: all authors. PI and NBZ accept full responsibility for the work and/or the conduct of the study, had access to the data, and controlled the decision to publish.

Funding This work was supported by the CDC through a cooperative agreement (grant 5U01CK000146-04), Wellcome Trust Programme Grant (Grant number
091909/Z/10/Z) and the MLW Programme Core Award (Grant 206454) from the Wellcome Trust.

Disclaimer The funders had no role in study design, data collection and analysis, decision to publish, or preparation of the manuscript. The findings and conclusions in this report are those of the authors and do not necessarily represent the official position of the Centers for Disease Control and Prevention, Atlanta USA.

Competing interests PI has received investigator-initiated research grant support from Bill \& Melinda Gates Foundation outside the scope of this work; NC has received research grant support and honoraria for participation in rotavirus vaccine advisory board meetings from GlaxoSmithKline Biologicals and from Takeda Pharmaceuticals outside the scope of this work; NF has received investigator-initiated research grant support from GlaxoSmithKline Biologicals and from Takeda Pharmaceuticals outside the scope of this work. NBZ has received investigator-initiated research grant support from GlaxoSmithKline Biologicals, Takeda Pharmaceuticals, Merck-Sharpe-Dohme and the Serum Institute of India, al outside the scope of this work. All other authors declare that they have no financial disclosures or competing interests.

Patient consent for publication Not applicable.

Ethics approval This study was approved by the University of Malawi College of Medicine Research Ethics Committee (P.05/13/1386) and by the University of Liverpool (RETH490). Participants gave informed consent to participate in the study before taking part.

Provenance and peer review Not commissioned; externally peer reviewed.

Data availability statement Data are available on reasonable request. Data is available on reasonable request to the corresponding author.

Supplemental material This content has been supplied by the author(s). It has not been vetted by BMJ Publishing Group Limited (BMJ) and may not have been peer-reviewed. Any opinions or recommendations discussed are solely those of the author(s) and are not endorsed by BMJ. BMJ disclaims all liability and responsibility arising from any reliance placed on the content. Where the content includes any translated material, BMJ does not warrant the accuracy and reliability of the translations (including but not limited to local regulations, clinical guidelines, terminology, drug names and drug dosages), and is not responsible for any error and/or omissions arising from translation and adaptation or otherwise.

Open access This is an open access article distributed in accordance with the Creative Commons Attribution 4.0 Unported (CC BY 4.0) license, which permits others to copy, redistribute, remix, transform and build upon this work for any purpose, provided the original work is properly cited, a link to the licence is given, and indication of whether changes were made. See: https://creativecommons.org/ licenses/by/4.0/.

\section{ORCID iD}

Pui-Ying Iroh Tam http://orcid.org/0000-0002-3682-8892

\section{REFERENCES}

1 UNAIDS. Malawi factsheet, 2019.

2 World Bank. Prevalence of stunting - Malawi, 2018.

3 WHO. Malawi maternal and child health data. Geneva, Switzerland: WHO, 2015.

4 WHO. Revised who classification and treatment of childhood pneumonia at health facilities. Geneva, Switzerland: WHO, 2014.

5 Iroh Tam P-Y, Musicha P, Kawaza K, et al. Emerging resistance to empiric antimicrobial regimens for pediatric bloodstream infections in Malawi (1998-2017). Clin Infect Dis 2019;69:61-8.

6 Landes M, van Lettow M, Chan AK, et al. Mortality and health outcomes of HIV-exposed and unexposed children in a PMTCT cohort in Malawi. PLoS One 2012;7:e47337.

7 Slogrove A, Reikie B, Naidoo S, et al. HIV-exposed uninfected infants are at increased risk for severe infections in the first year of life. $J$ Trop Pediatr 2012;58:505-8.

8 Slogrove AL, Goetghebuer T, Cotton MF, et al. Pattern of infectious morbidity in HIV-exposed uninfected infants and children. Front Immunol 2016;7:164.

9 Zar HJ, Barnett W, Stadler A, et al. Aetiology of childhood pneumonia in a well vaccinated South African birth cohort: a nested case-control study of the Drakenstein child health study. Lancet Respir Med 2016;4:463-72.

10 Malawi Ministry of Health. Clinical management of HIV in adults and children. Malawi, 2011. 
11 WHO. Handbook IMCl. Integrated management of childhood illness. Geneva, Switzerland: World Health Organization, 2000.

12 Emukule GO, McMorrow M, Ulloa C, et al. Predicting mortality among hospitalized children with respiratory illness in Western Kenya, 2009-2012. PLoS One 2014;9:e92968.

13 Hooli S, Colbourn T, Lufesi N, et al. Predicting hospitalised paediatric pneumonia mortality risk: an external validation of RISC and mRISC, and local tool development (RISC-Malawi) from Malawi. PLoS One 2016;11:e0168126.

14 Reed C, Madhi SA, Klugman KP, et al. Development of the respiratory index of severity in children (RISC) score among young children with respiratory infections in South Africa. PLoS One 2012;7:e27793.

15 Deardorff KV, McCollum ED, Ginsburg AS. Pneumonia risk stratification scores for children in low-resource settings: a systematic literature review. Pediatr Infect Dis J 2018;37:743-8.
16 Gallagher KE, Knoll MD, Prosperi C, et al. The predictive performance of a pneumonia severity score in human immunodeficiency virus-negative children presenting to hospital in 7 low- and middle-income countries. Clin Infect Dis 2020;70:1050-7.

17 Justicia-Grande AJ, Pardo-Seco J, Cebey-López M, et al. Development and validation of a new clinical scale for infants with acute respiratory infection: the ReSVinet scale. PLoS One 2016;11:e0157665.

18 Iroh Tam P-Y, Sadoh AE, Obaro SK. A meta-analysis of antimicrobial susceptibility profiles for pneumococcal pneumonia in sub-Saharan Africa. Paediatr Int Child Health 2018;38:7-15.

19 Iroh Tam P-Y. The challenge and opportunity of pediatric antimicrobial stewardship in low resource settings. $J$ Trop Pediatr 2020;66:1-3. 